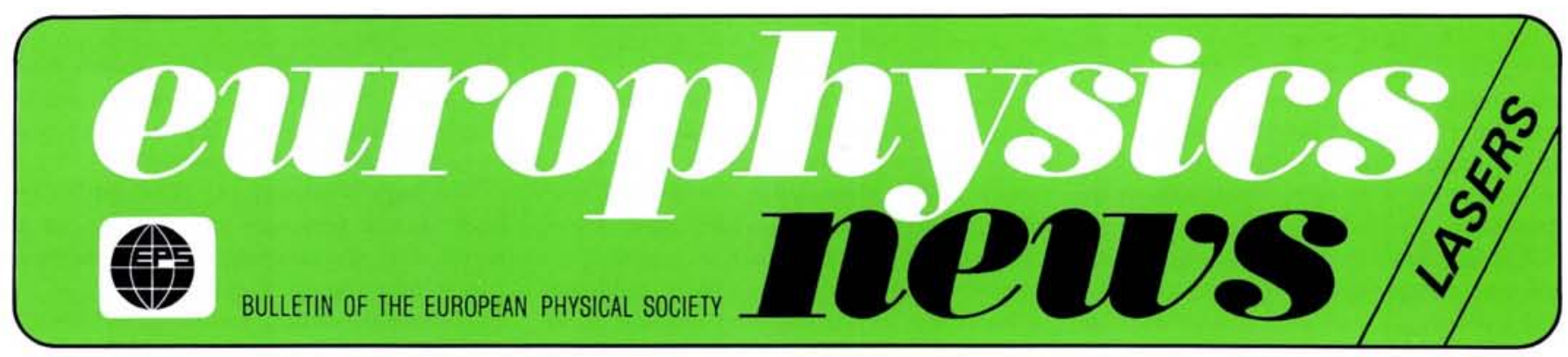

J.A.

Volume 12

Number 7

July 1981

\title{
the laser
}

Twenty one years since their discovery, lasers are still in full development. Their contribution to both science and technology has already been remarkable, and they are finding wide application in many fields.

\section{Physical Principles and Historical Review}

\section{O. Svelto, Milan}

(Quantum Electronics and Electronic Instrumentation Centre of the (NR)

\section{Origin of the Laser}

Lasers rely essentially on the process of stimulated emission, in which an excited species is forced to release at least part of its energy to an incoming electromagnetic wave (e.m.) of appropriate wavelength. This phenomenon was already predicted by Einstein in 1917 in his famous paper "Zur Quantenmechanik der Strahlung". It took more than thirty years, however, for people to realize that, in a non-thermal equilibrium, amplification and coherent generation of electromagnetic radiation was possible by stimulated emission.

One of the first proposals to this end, was contained in a patent that was granted in 1951 to V.A. Fabrikant, of the Moscow Power Institute, but being published in the official Soviet patent organ, it became available only in 1959. In 1954-1955, N.G. Basov and A.M. Prokhorov, of the Lebedev Institute in Moscow, in a series of papers, published a proposal and detailed calculations for a microwave oscillator based on stimulated emission. Meanwhile, in 1954 , a generator of this kind was operated, for the first time, by C.H. Townes and co-workers, at Columbia University, using as the emitting species, a prepared beam of ammonia molecules. Townes, who had been working along these lines since 1951, coined the term "Maser", an acronym for Microwave Amplification by Stimulated Emission of Radiation. For their fundamental work, Basov, Prokhorov and Townes were awarded the Nobel Prize in Physics in 1964. The Maser can properly be considered the closest precursor of the Laser.
Initial Laser Development

The extension of the maser principle to the optical or infrared region of the e.m. spectrum was proposed in 1958 by A.L. Schawlow (Bell Telephone Laboratories) and Townes although in 1977, the U.S. Patent Office granted several rights concerning the invention of the laser to G. Gould. Gould's works, however, were never published, and the corresponding results were not made directly available to the scientific community. Perhaps, one of the most stimulating aspects of the paper of Schawlow and Townes was the calculation, for a general atomic system, of the minimum rate at which atoms or molecules need to be raised to the excited state to give net stimulated emission.

A second important aspect of the paper was the proposal to use an open resonator made of two plane parallel mirrors (the Fabry-Perot resonator), to trap the radiation emitted by the atoms. In this, the resonator modes that could oscillate were greatly reduced, corresponding to those of an e.m. wave propagating in a direction nearly orthogonal to the two mirrors. The same proposal was, independently and at about the same time, also considered by Prokhorov, for the construction of a generator in the millimeter wavelength range.

The proposal of Schawlow and Townes aroused the interest of many laboratories, both in the academic and in the industrial world, and in June 1960, T.H. Maiman, at Hughes Labs., was the first to obtain stimulated emission in the visible spectrum.
Maiman used a pulsed high-power flash tube as the pumping source, and a ruby rod as the active material. The two end faces of the ruby rod were made parallel to one another and then suitably coated so forming the two mirrors of a Fabry-Perot resonator. The onset of stimulated emission was observed through a shortening of the fluorescence lifetime and a decrease in its bandwidth. By analogy with the term Maser, Maiman coined the word LASER for his device, as an acronym for "Light Amplification by Stimulated Emission of Radiation", a word also used by Gould in his early works, and which then gained acceptance over "optical maser", employed by some other early workers in the field. Shortly afterwards, Schawlow, Collins and co-workers at Bell Laboratories, observed the onset of coherent oscillations with a similar set-up and they were the first to point out such fundamental properties as directionality, coherence, and relaxation oscillations. A few months later. P.P. Sorokin and co-workers, at the IBM Research Centre, operated two other optically-pumped solid-state lasers, based on $\mathrm{U}^{3+}$ and $\mathrm{Sm}^{2+}$ in $\mathrm{CaF}_{2}$. When cooled to

\section{Contents}

Special Issue on Lasers

Physical Principles and Historical Review

Applications to Photochemistry

Isotope Separation

Industrial Applications

Optical Storage on Discs

Plasma Diagnostics

Applications to Biology

and Medicine

ECAP

Society News

1
4
5
10
11
12
14
15
16

4

5

12

14

15

16 
cryogenic temperatures, both lasers operated on the four-level principle (as opposed to ruby which works as a three-level laser), thus calling for two or three orders of magnitude less pump energy from the flash-tube.

The last breakthrough came in December with the achievement by A. Javan, at Bell Laboratories, of laser operation in the near infrared from a helium-neon gas mixture pumped by an electric discharge. This was the first gas laser in history. The active material was neon with helium added to improve the pumping efficiency (by a process known as resonant energy transfer), as already proposed by Javan in 1959. An important aspect of this laser was that, for the first time, continuous wave (cw) operation was demonstrated. Moreover, and even more important perhaps, it was shown that stimulated emission in the optical or infrared region could be achieved in a completely different medium and with a different excitation mechanism.

\section{Later Laser Development}

These initial discoveries aroused enormous interest in the scientific community, and many laboratories throughout the world started research in the laser field.

\section{Solid-State Ion Lasers}

The invention of the ruby laser $\left(\mathrm{Cr}^{3+}\right.$ in $\mathrm{Al}_{2} \mathrm{O}_{3}$ ) and of the lasers based on $\mathrm{U}^{3+}$ and $\mathrm{Sm}^{2+}$ stimulated intensive research effort to find new solid-state lasers. Soon, it became clear that the ions to be looked at should belong to a transition series of the periodic table (as $\mathrm{Cr}, \mathrm{U}$, and $\mathrm{Sm}$ ). The minimum threshold pump power per unit volume of the material, $d P_{t h} / d V$, can be shown (for a four-level laser) to be given by:

$d P_{t h} / d V=h v_{p} \gamma / \sigma l \tau$

where $v_{p}$ is the frequency of the pump transition, $\gamma$ the resonator loss per pass, $l$ the length of the active material, $\sigma$ the crosssection, and $\tau$ the lifetime of the laser transition. From (1) it is apparent that good candidates for laser action are transitions with high values of the $\sigma \tau$ product. Ions of the transition series have, indeed, unfilled inner-shell transitions that are sharp (i.e. with relatively high values of $\sigma, \sim 10^{-18}$ $\mathrm{cm}^{2}$ ) and long-lived (i.e. with values of $\tau$ in the ms range, as they are electric-dipole forbidden).

A large number of solid-state ion lasers emitting in the visible and the near infrared were thus discovered in the sixties. In particular, $\mathrm{Nd}^{3+}$ was soon recognized as one of the best ions (Snitzer and Johnson, 1961). As it can work on the four-level scheme at room temperature, $\mathrm{Nd}^{3+}$ has become the most widely-used opticallypumped solid-state laser with as host, a crystal of YAG, acronym for Yittium Aluminum Garnet.

\section{Semiconductor Lasers}

The possibility of obtaining laser action in a semiconductor was considered quite early, notably by N. Basov, a former student of Prokhorov who, since 1955, had established at Lebedev an independent laboratory. To begin with however, proposals were rarely made with sufficient knowledge of the required material parameters. Thus, at the second Conference on Quantum Electronics (Berkeley, 1961), attention was still directed towards indirect gap semiconductors such as $\mathrm{Ge}$ or Si. Only about a year later did it become clear that laser action in germanium or silicon could not be achieved on account of free-carrier absorption.

At the same time, it became apparent that this difficulty was of much less importance in direct-gap semiconductors, such as III-V compounds. Gallium arsenide, which was already under development for other applications, then became the most likely candidate, so it was not surprising that the achievement of laser action in a forward-biased GaAs p-n diode was announced, at the end of 1962 , by three research groups working at IBM, General Electric Corp., and Lincoln Laboratory. In fact, papers from the first two laboratories were published on the same day ( $1 \mathrm{Nov}$.) by two different scientific journals, while the work of the third group was published shortly afterwards.

Impressive technological progress has been made in $\mathrm{GaAs}$ diode technology since 1962. A breakthrough in this development was the introduction (in 1968) of the (AIGa)As - GaAs single heterojunction laser, soon followed by the double heterojunction variant. In the latter case, the active material, a thin $(0.1-0.5 \mu \mathrm{m})$ layer of $\mathrm{GaAs}$ forms two heterojunctions with respectively $\mathrm{p}$ - and $\mathrm{n}$-type (AIGa)As. Unlike the original $\mathrm{GaAs}$ laser, laser action here is strictly confined to the (GaAs) active materials, and the losses due to free-carrier absorption are drastically reduced. This has resulted in a spectacular decrease, by more than two orders of magnitude, of the threshold current density, enabling semiconductor lasers to be operated $\mathrm{cw}$ at room temperature and thus opening up wide application possibilities (notably in the field of optical communications).

\section{The $\mathrm{CO}_{2}$ Laser}

The $\mathrm{CO}_{2}$ laser was invented early in 1964 by C.K.N. Patel and co-workers at Bell Laboratories. This laser uses a mixture of $\mathrm{CO}_{2}, \mathrm{~N}_{2}$ and $\mathrm{He}$, and oscillation takes place on a roto-vibrational transition of the $\mathrm{CO}_{2}$ molecule, while $\mathrm{N}_{2}$ and $\mathrm{He}$ are added to improve efficiency. More precisely, the most common laser transition at $\lambda=10.6 \mu \mathrm{m}$ occurs as a result of the molecule's transition between the first excited states (asymmetric and symmetric stretching). It is one of the most efficient and most powerful lasers so far available. Indeed, in less than two years (in 1965) Patel was already reporting an output power of $100 \mathrm{~W}$, which was quite impressive for that time.

The high efficiency $(\sim 20 \%)$ of a $\mathrm{CO}_{2}$ laser is the fortunate combination of a series of circumstances that are seldom met in other types of laser, namely:

(i) the electron impact excitation of the $\mathrm{CO}_{2}$ asymmetric stretching occurs with a very high efficiency;

(ii) the electron impact excitation of the $\mathrm{N}_{2}$ vibration also occurs with a very high efficiency, and the corresponding energy is then easily transferred to the asymmetric stretching mode of the $\mathrm{CO}_{2}$ molecule (by resonant energy transfer);

(iii) helium is very effective in depleting the lower laser level, thus allowing for an effective four-level operation of the $\mathrm{CO}_{2}$ molecule:

(iv) the energy of the emitted photon is a large fraction $(\sim 40 \%)$ of that needed to excite the asymmetric stretching vibration.

It is not therefore surprising that intensive work has been done on this type of laser since 1965, leading to the achievement of spectacular values for the output power (more than $100 \mathrm{~kW}$ in $\mathrm{cW}$ operation!). Several geometrical configurations, in which both the flow of the gas mixture and the flow of the discharge electrons occur either along the laser resonator or across it, have been developed.

A particular type of $\mathrm{CO}_{2}$ laser deserves special mention. In this, the population inversion is not produced by an electrical discharge but by supersonic expansion of a gas mixture (containing $\mathrm{CO}_{2}$ ) initially heated to a high temperature. The key point here is that the lifetime of the upper state of the $\mathrm{CO}_{2}$ molecule is longer than that of the lower state, a condition that must always be met to achieve $\mathrm{cw}$ operation. Consequently, in the cooling process arising from the expansion, the population of the upper laser level decays more slowly than that of the lower laser level. A population inversion, leading to laser action, can thus be produced downstream from the expansion region. The idea of creating a population inversion by rapid heating or cooling a molecular system was suggested by Basov and Oraevskii already in 1962 . Subsequently, Hurle and Hertzberg (1965) suggested that this could be obtained in the rapid, nonequilibrium expansion of an initially hot gas through a supersonic nozzle. The first $\mathrm{CO}_{2}$ gasdynamic laser was then operated by Kantrowitz et al. at Avco Everett Research Laboratory in 1966.

\section{Noble Gas Ion Laser}

After Javan's discovery of laser action in the infrared transition of the neon atom, most noble gases in their neutral states were made to oscilla:e. Almost all oscillate in the infrared, the only notable exception being the $\mathrm{He}-\mathrm{Ne}$ laser which oscillates on 
its now widely-used red $(\lambda=633 \mathrm{~nm})$ transition (discovered in 1962 by White). The development of a gas laser working in the visible (and notably in the blue-green region of the spectrum) was therefore thought to be highly desirable.

A solution lay in the use of ions of the noble gases where the scale of energy levels is expanded, the ionization potential being about twice that of the neutral atom. Consequently, the transitions of an ionized species can be expected to occur at shorter wavelengths.

Laser action was indeed demonstrated almost simultaneously in 1964 by Bridges, Converter et al., and Bennet et al., on several blue and green lines of the $\mathrm{Ar}^{+}$ spectrum. Another notable laser of this class is the $\mathrm{Kr}^{+}$laser, which has a powerful oscillation in the red. Population inversion in an ion laser occurs through two successive collisions of the species with the electrons in the discharge. The first produces an ion from the neutral atom, while the second excites this ion. Although widely used, these lasers have, nevertheless, very poor efficiency $\left(\sim 10^{-3}\right)$, partly because of this two-step process. A second reason is that, with for instance $\mathrm{Ar}^{+}$, the $\mathrm{Ar}^{+}$ions must be pumped to a very high energy ( $>35 \mathrm{eV}$ above the ground level of the neutral atom) to achieve laser action and give photons of only about $2 \mathrm{eV}$.

\section{Chemical Lasers}

In a chemical laser, the population inversion is produced by a chemical reaction, the fascinating possibility of which was considered quite early. Proposed by Polanyi in 1961, it was first demonstrated by Kasper and Pimentel in 1964. No substantial effort however, was devoted to the subject until after 1969 when the first purely chemical laser, in which bottled gases were directly mixed, was demonstrated. Chemical lasers usually involve a chemical reaction between gaseous elements, in which a large proportion of the reaction energy is often left in the form of vibrational energy of the molecules. Transitions are therefore often of the vibrationalrotational type, the only notable exception being the iodine molecular photodissociation laser.

The reason why, in some cases, a chemical reaction leads to vibrationally-excited molecules can be understood by considering one of the most classic reactions used, namely

$$
\mathrm{F}+\mathrm{H}_{2} \rightarrow \mathrm{HF}^{*}+\mathrm{H}
$$

This leads to vibrationally-excited HF, which then produces laser action. Because of the high electron affinity of $\mathrm{F}$, the $\mathrm{F}-\mathrm{H}_{2}$ interaction is strongly attractive at large distances, leading to a considerable polarization of the $\mathrm{H}_{2}$ charge distribution. The HF bond can therefore be formed before $F$ has reached the inter-nuclear separation, appropriate to the HF ground electronic state. Thus, there is a considerable probability that HF will be found, after reaction, at a distance greater than its equilibrium value. This leads, classically, to vibrational excitation.

The above argument points to one of the main problems associated with chemical lasers: to obtain vibrationally-excited molecules, highly reactive species must be involved, the handling of which constitutes a serious limitation in their development.

\section{Dye Lasers}

There is a wide variety of dyes that, when dissolved in an appropriate solvent and excited by light of the appropriate wavelength, show very intense fluorescence. It is therefore natural to consider such dyes as good candidates for laser action when pumped by a suitable optical source. Despite this, the development of dye lasers took place rather late ( $P$. Sorokin, 1966). One of the reasons can be understood by considering Eq. (1). For a typical dye, we have $\sigma \cong 10^{-16} \mathrm{~cm}^{2}$ but $\tau \cong$ $10^{-8} \mathrm{~s}$. Thus, the product $\sigma \tau$ is, typically, three orders of magnitude less than for $\mathrm{Nd}^{3+}$, for instance. It must be expected therefore, that the threshold pump energy per unit volume $d P_{t h} / d V$ will be much greater than for Nd:YAG. This explains why, despite much effort, dye lasers can still only work in pulsed operation when pumped by an incoherent light source, which must have a very high intensity. Dye lasers can however, operate $\mathrm{cw}$ when pumped by another laser (typically, $\mathrm{Ar}^{+}$or $\mathrm{Kr}^{+}$), and they are now widely used in scientific applications, mainly because they can give an output beam of wavelength that can be continuously tuned throughout the visible range.

\section{Excimer Lasers}

An excimer is a molecule which only exists (i.e. is bound) in the excited state. An excimer laser, such as that based on $\mathrm{Xe}_{2}^{*}$, the first to operate (Basov et al. 1971), thus involves (often) a transition between the upper (bound) state to the lower (unbound) state of the molecule. The lower state is empty (the two atoms separate quickly upon dissociation) and this enables the laser to operate on the four-level principle.

A particular class of excimer laser which strictly speaking, should not be called excimer, since they involve the bonding of unlike partners, comprises the gas-halide excimers (first demonstrated by several groups in the U.S. in 1975). In the excited state, a rare gas atom reacts easily with halogens to form a (hetero-) excimer, chemically similar to an alkali halide, the bonding of which is predominantly ionic in character.

Rare-gas-halide excimer lasers are now widely used, since they are the most powerful laser sources at several wavelengths in the UV.

\section{Molecular Beam Epitaxy}

Arthur D. Little, inc. invites applications for a position in the molecular beam epitaxy laboratory in the physical systems research section.

Applicants must be qualified to carry operational responsibility for the laboratory.

They must have demonstrated their ability to perform independent research and be prepared to take part in innovative applications.

Those with experience and interest in working on analytical and electrical characterization will be given preference.

Please respond by resume only to:

L.J. Clevenger, jr.,

Arthur D. Little inc., Acorn Park,

Cambridge, Massachusetts, USA 2140.

An equal opportunity employer $m / f / h /$.

\section{Towards the X-Ray Region}

To obtain oscillation in the UV or VUV, vibronic transitions of suitable molecules ( notably $\mathrm{N}_{2}, \lambda=337.1 \mathrm{~nm}$ and $\mathrm{H}_{2}, \lambda=160$ and $116 \mathrm{~nm}$ ) have been used, while at still shorter wavelengths, down to the X-ray region, transitions of multiple ionized elements (e.g. $\mathrm{C}^{5+}$ ) could be employed. However, no one has been able to operate a laser in the $\mathrm{X}$-ray region (even in the soft $X$-ray region), and the $\mathrm{H}_{2}$-laser with its $\lambda=$ $116 \mathrm{~nm}$ transition still remains the shortestwavelength laser available. The reason for this can again be understood by considering Eq. (1). At the high frequencies $v$ of the $X$-ray region, the lifetime $\tau$ is given by the radiative lifetime $\tau_{r}$, which is proportional to $1 / v^{3}$, and $\sigma$ becomes proportional to $1 / v^{2}$. Taking $v_{\mathrm{p}} \cong v$ in Eq. (1) we thus see that $d P_{t h} / d V$, as a function of the transition frequency, is expected to be proportional to $v^{6}$ and thus to increase considerably.

\section{Conclusions}

As can be seen from this short review on laser development, the physical principles involved in each laser (starting from the general idea of the stimulated emission process) are quite different from one another. These differences and the corresponding difficulties associated with their technical realisation may often explain the different histories of the various classes of laser. However, the differences in operating principle and in output beam performance are also among the most fascinating aspects.

Lasers, once called (by non-believers) a bright solution in search of a problem, can nowadays be considered the bright solution to a wide variety of problems, both in science and in technology. 